\title{
Cost-Effectiveness Modelling of Sequential Biologic Strategies for the Treatment of Moderate to Severe Rheumatoid Arthritis in Finland
} \author{
K. Sennfalt ${ }^{6}$ and A. Beresniak ${ }^{*}, 7,8$ \\ ${ }^{1}$ Lappeenranta Central Hospital, Lappeenranta, Finland \\ ${ }^{2}$ Seinäjoki Central Hospital, Seinäjoki, Finland \\ ${ }^{3}$ Rheumatism Foundation Hospital, Heinola, Finland \\ ${ }^{4}$ Helsinki University Central Hospital, Helsinki, Finland \\ ${ }^{5}$ Bristol-Myers Squibb, Helsinki, Finland \\ ${ }^{6}$ Bristol-Myers Squibb, Stockholm, Sweden \\ ${ }^{7}$ Data Mining International, Geneva, Switzerland \\ ${ }^{8}$ Paris-Descartes University, Paris, France
}

K. Puolakka ${ }^{1}$, H. Blåfield ${ }^{2}$, M. Kauppi $^{3}$, R. Luosujärvi ${ }^{4}$, R. Peltomaa ${ }^{4}$, T. Leikola-Pelho ${ }^{5}$,

\begin{abstract}
Objective: The main objective was to compare the cost-effectiveness of therapeutic options in moderate or severe rheumatoid arthritis (RA) when a clinical response to a first TNF-blocker, either etanercept (ETA), adalimumab (ADA), or infliximab (INF), is insufficient.

Methods: Effectiveness criteria were defined as remission (RS), low disease activity (LDAS), and moderate to high disease activity (MHDAS). Cost-effectiveness was derived as cost per day in RS and in LDAS using simulation modelling to assess six sequential biologic strategies over 2 years. Each sequential treatment strategy was composed of three biologic agents and included a first anti-TNF agent, ETA, ADA or INF, followed by either abatacept (ABA) or rituximab (RTX) as a second therapeutic option in case of an insufficient response, followed by another anti-TNF agent in case of further insufficient response.

Results: Over two years and taking into account biologic costs, the following estimated mean costs per day in RS and LDAS were respectively of $€ 829$ and $€ 428$ for the biologic sequence composed of ADA-ABA-ETA, $€ 1292$ and $€ 516$ for the sequence ADA-RTX-ETA, $€ 829$ and $€ 429$ for the sequence ETA-ABA-ADA, $€ 1292$ and $€ 517$ for the sequence ETARTX-ADA, $€ 840$ and $€ 434$ for the sequence INF-ABA-ETA, and $€ 1309$ and $€ 523$ for the sequence INF-RTX-ETA.

Conclusion: The treatment sequences including ABA as the second biologic option appear more cost-effective than those including RTX in a patients with moderate to severe RA and an insufficient response to a first anti-TNF agent.
\end{abstract}

Keywords: Cost-effectiveness, rheumatoid arthritis, biologics, modelling.

\section{INTRODUCTION}

Rheumatoid Arthritis (RA) is a common, chronic inflammatory disease that produces high rates of morbidity and disability, as well as increased mortality, and poses a significant challenge and a substantial economic burden both on patients and on the healthcare system. According to a recent study, the incidence of RA in Finland is 44.5 per 100 '000 individuals over 16 years of age [1].

Therapeutic guidelines recommend that RA treatment be initiated with traditional disease-modifying antirheumatic drugs (tDMARDs), either with one DMARD or with a combination of DMARDs, methotrexate being the "anchor

*Address correspondence to this author at the Data Mining International, Route de l'Aéroport, 29-31, Case Postale 221, CH-1215 Geneva 15, Switzerland; Tel: + 412279934 00; Fax: + 412278838 50;

E-mail: aberesniak@datamining-international.com drug" and current standard of care. In case of treatment failure to tDMARDs, the new biologic agents (bDMARDs) offer further therapeutic treatment options for patients with moderate to severe RA. The anti-tumor necrosis factor (antiTNF) agents are usually the first bDMARDs to be prescribed. In case of an insufficient response to a first antiTNF agent, the TNF-inadequate responders (IR) can then either be switched to another anti-TNF agent or be treated with newer biologic agents abatacept (ABA) or rituximab (RTX), both having distinct and different mechanisms of action from the anti-TNF agents [2].

Although RA sequential biological treatments represent current medical practices, no comparative clinical trials are available to assess the clinical efficacy and cost-effectiveness of different bDMARDs used in sequence. This may be explained by the methodological challenges and the prohibitive costs of conducting such complex clinical trials. However, using the results from placebo-controlled clinical 
trials for each bDMARDS, decision analytic models can be developed and simulation modelling can be used to compare different treatment sequences. Such models consist of robust mathematic and probabilistic formulas linking published clinical evidence and costs in clinical environments modelled to reflect local medical practices. This approach is aligned with the Outcome Measures in Rheumatology Clinical Trials (OMERACT) recommendations [3]. Results generated from advanced simulation modelling thus provide unique information on the expected effectiveness, overall costs, and cost-effectiveness of different biologic strategies in order to assist clinical decision-making, as well as resource allocation decisions from public health officials.

In Finland, biologic agents can be prescribed in patients with moderate or severe RA. Hence, the objective of this advanced modelling study is to assess the effectiveness, costs and cost-effectiveness of different sequential biologic treatment strategies for managing moderate to severe RA in patients with an insufficient response to a first anti-TNF agent in Finland.

\section{MATERIAL AND METHODOLOGY}

\section{Effectiveness Inputs}

Two clinically relevant success endpoints were chosen as effectiveness criteria [4]. Patients achieving a Disease Activity Score (DAS28) $<2.6$ were defined to be in remission state (RS), whereas patients achieving a score $\leq 3.2$ were defined to be in low disease activity state (LDAS), and those with a score $>3.2$ were defined to be in moderate to high disease activity state (MHDAS).

Effectiveness estimates of biological therapies in antiTNF inadequate responders were derived directly from published clinical trials at the time of model development (Tables 1 and 2). Data from randomised controlled clinical trials were used if they reported DAS status expressed in remission or LDAS rates at 6 months. Thus, the ATTAIN trial and long-term extension study were used for ABA $[5,6]$, the open label ReAct trial for anti-TNF agents [7] and the REFLEX trial and open-label extension analysis for RTX $[8,9]$. The ATTAIN and REFLEX clinical trials were deemed comparable in terms of patients baseline characteristics (age, gender, disease duration and DAS28 score at baseline). In absence of published effectiveness data for INF or ETA at time of model development, the results of the ReAct trial, an open label study [7], were used as best surrogate evidence for all anti-TNF agents used as third biologic option. This was reinforced by the fact that although the ReAct study specifically concerns ADA, the results correlate well with those of the Karlsson JA study [10] which studied treatment response to anti-TNF switches, regardless of the anti-TNF agents used. For the purpose of modelling RTX re-treatment intervals, as most patients who received additional courses during RTX clinical trials (where the need for repeated courses were at physician's discretion based on specific response criteria) did so 24 weeks after the previous course, and as none were re-treated sooner than 16 weeks (RTX US Product monograph), the model set RTX retreatment intervals at 6 months in order to sustain a constant DAS28 response over time. The data sources are summarized in Tables $\mathbf{1}$ and $\mathbf{2}$.

\section{Costs Inputs}

Resource utilization for these three disease activity states (RS, LDAS and MHDAS) was assessed by an expert panel of clinical rheumatologists to reflect current medical practices in Finland. The panel described ten RA resource utilization items: specialist visits, general practionners visits, nurse visits, rehabilitation care, laboratory tests, $\mathrm{x}$-ray, hospitalization, ortheses (insoles, splints), devices for daily activities, and potential household workers. Unit costs from the public payer perspective were collected and simulated using distribution ranges for each item. Using a specific costing model, a computation of all costs distributions was performed to estimate the overall direct medical costs over 6 months. Similar costing methods were used and published for other European countries [11]. Biologic drug costs were estimated based on the 2009 price lists and recommended dosing in Finland. All direct medical costs were estimated per 6-month intervals.

\section{Model Structure}

Simulation models represent a type of decision trees which allow to compute variable distributions. Twelve separate models were developed to simulate 6 sequences composed of 3 biologic agents using two "success"

Table 1. Summary of Effectiveness Probabilities (Percentage of Patients Achieving LDAS)

\begin{tabular}{|c|c|c|c|}
\hline Biologic Agent & & \%LDAS & Source \\
\hline Abatacept after IR to anti-TNF therapy & $\begin{array}{l}\text { Induction -Month } 12 \\
\text { Maintenance } \\
\text { Month } 18 \\
\text { Month } 24\end{array}$ & $\begin{array}{c}18.3 \% \\
24.2 \% \\
28 \%\end{array}$ & $\begin{array}{l}\text { ATTAIN + LTE study (Genovese 2007) } \\
\text { ATTAIN + LTE study (Genovese 2007) } \\
\text { ATTAIN + LTE study (Genovese 2007) }\end{array}$ \\
\hline Anti-TNF agents & $\begin{array}{l}\text { Induction - Month } 18 \\
\text { Maintenance - Month } 24\end{array}$ & $\begin{array}{c}11 \% \\
21.5 \%\end{array}$ & $\begin{array}{l}\text { REACT trial (Bombardieri 2007) } \\
\text { ATTAIN reanalysis afterIR to } 2 \text { anti-TNF agents }\end{array}$ \\
\hline Rituximab after IR to anti-TNF therapy & $\begin{array}{l}\text { Induction - Month } 12 \\
\text { Maintenance } \\
\text { Month } 18 \\
\text { Month } 24\end{array}$ & $\begin{array}{l}13 \% \\
25 \% \\
29 \%\end{array}$ & $\begin{array}{l}\text { REFLEX + LTE study (Keystone 2007) } \\
\text { REFLEX + LTE study (Keystone 2007) } \\
\text { Keystone (EULAR 2007) }\end{array}$ \\
\hline DMARDS & Month 24 & $5 \%$ & Clinical experts opinion \\
\hline
\end{tabular}

IR = Insufficient Response. 
Table 2. Summary of Effectiveness Probabilities (Percentage of Patients Achieving RS)

\begin{tabular}{|c|c|c|c|}
\hline Abatacept after IR to anti-TNF therapy & $\begin{array}{l}\text { Induction -Month } 12 \\
\text { Maintenance } \\
\text { Month } 18 \\
\text { Month } 24\end{array}$ & $\begin{array}{l}11.1 \% \\
13.9 \% \\
17.1 \%\end{array}$ & $\begin{array}{l}\text { ATTAIN + LTE study (Genovese 2007) } \\
\text { ATTAIN + LTE study (Genovese 2007) } \\
\text { ATTAIN + LTE study (Genovese 2007) }\end{array}$ \\
\hline Anti-TNF agents & $\begin{array}{l}\text { Induction - Month } 18 \\
\text { Maintenance - Month } 24\end{array}$ & $\begin{array}{c}4 \% \\
14.4 \%\end{array}$ & $\begin{array}{l}\text { REACT trial (Bombardieri 2007) } \\
\text { ATTAIN reanalysis after IR to } 2 \text { anti-TNF agents }\end{array}$ \\
\hline Rituximab after IR to anti-TNF therapy & $\begin{array}{l}\text { Induction - Month } 12 \\
\text { Maintenance } \\
\text { Month } 18 \\
\text { Month } 24\end{array}$ & $\begin{array}{l}6 \% \\
13 \% \\
12 \%\end{array}$ & $\begin{array}{l}\text { REFLEX + LTE study (Keystone 2007) } \\
\text { REFLEX + LTE study (Keystone 2007) } \\
\text { Keystone (EULAR 2007) }\end{array}$ \\
\hline DMARDS & Month 24 & $1 \%$ & Clinical experts opinion \\
\hline
\end{tabular}

endpoints (RS or LDAS)[4,12-14]. In the simulation models developed for this study, each new biologic drug introduced in the sequence is maintained as long as the clinical response is adequate, i.e., achieving at least LDAS. In case of an insufficient response, treatment switches are allowed at each 6-month time points. In order to reflect a population of anti$\mathrm{TNF}$ inadequate responders, all the sequences assume an insufficient response to the first anti-TNF agent appearing in each sequence, meaning that the first anti-TNF was not efficacious in all patients at the end of the first 6 months. The following sequences were modelled accordingly: Sequence A: ADA-ABA-ETA; Sequence B: ADA-RTXETA; Sequence C: ETA-ABA-ADA; Sequence D: ETARTX-ADA; Sequence E : INF-ABA-ETA; Sequence F: INFRTX-ETA.

In sequence $\mathrm{A}$ and $\mathrm{B}$, the first anti-TNF is ADA. Hence, this means that in sequence $\mathrm{A}$, patients are switched to ABA after the first 6-month interval. In case of insufficient response to ABA, ETA is then used as the third potential biologic agent (Fig. 1). In sequence B, patients are first switched to RTX, after which ETA is used in case of an insufficient response to RTX. Similarly, sequences C and D assume an insufficient response to ETA, after which patients are switched to either ABA (sequence C) or RTX (sequence D), and further switched to ADA in case of further insufficient response to these agents. Sequences $\mathrm{E}$ and $\mathrm{F}$ assume an insufficient response to INF. In sequence $\mathrm{E}$, patients are then switched to ABA, and then to ETA in case of further insufficient response, while in sequence $\mathrm{F}$, patients are switched to RTX, and ultimately to ETA in case of further insufficient response. The model assumed that patients failing three biologic options would be maintained on tDMARDS with a residual effectiveness of achieving RS and LDAS estimated at $1 \%$ and $5 \%$, respectively.

Mean values and standard deviations of the model outputs - costs, effectiveness, and mean cost-effectiveness over 2 years - were generated by 10'000 Monte-Carlo simulations. This approach, also called "probabilistic sensitivity analysis", allows to screen all possible values of a given parameter according to its distribution and to recalculate the results with standard deviations. Two-group means tests with known variances were performed to calculate potential significant differences between treatment strategies. As no Incremental cost-effectiveness ratio (ICER between two strategies = difference in costs / difference in effectiveness ) thresholds exist, the results are expressed as mean cost-effectiveness values to allow comparisons between the different treatment sequences. In this particular context, ICERs would otherwise be extremely difficult if not impossible to interpret.

\section{RESULTS}

Direct medical costs of managing RA in Finland (excluding the cost of biological therapies) were estimated at $€ 527$ ( $\mathrm{SD}=123$ ) over 6 months for patients achieving RS, $€ 2724(\mathrm{SD}=1131)$ for patients achieving LDAS, and $€ 5600$ $(\mathrm{SD}=2243)$ for patients in MHDAS. Hence, achieving LDAS or remission was associated with lower medical costs (Fig. 1). Key costs drivers were hospitalization and medical visits.

Costs per day in RS or per day in LDAS, were respectively $€ 829(\mathrm{SD}=85)$ and $€ 428 \quad(\mathrm{SD}=45)$ for the biologic sequence A (ADA-ABA-ETA), €1292 (SD=136) and $€ 516$ ( $\mathrm{SD}=56$ ) for sequence $\mathrm{B}$ (ADA-RTX-ETA), $€ 829$ $(\mathrm{SD}=85)$ and $€ 429(\mathrm{SD}=46)$ for sequence $\mathrm{C}$ (ETA-ABA$\mathrm{ADA}), € 1292(\mathrm{SD}=136)$ and $€ 517(\mathrm{SD}=56)$ for sequence $\mathrm{D}$ (ETA-RTX-ADA), $€ 840$ ( $\mathrm{SD}=85$ ) and $€ 434(\mathrm{SD}=45)$ for the sequence $\mathrm{E}$ (INF-ABA-ETA), and $€ 1309$ (SD=136) and $€ 523$ ( $\mathrm{SD}=56$ ) for sequence F (INF-RTX-ETA). Significant differences in cost-effectiveness ratios were observed between sequences A and B $(p<0.01), C$ and D $(p<0.01)$, as well as $E$ and $F(p<0.01)$ favouring sequences containing ABA vs RTX.

\section{DISCUSSION}

After taking into account all relevant clinical data and the Finnish public health care system perspective and constraints, our models generated original results showing that sequences including ABA appear more efficacious and cost-effective than similar sequences including RTX for patients with moderate to severe RA, who have experienced insufficient response to a first anti-TNF agent. Our results also clearly establish the relation between disease activity and medical resources. One of the most important issues in the creation of valid medico-economic models is the use of clinical effectiveness endpoints that are clinically meaningful and consistent across different settings. Selecting objective 


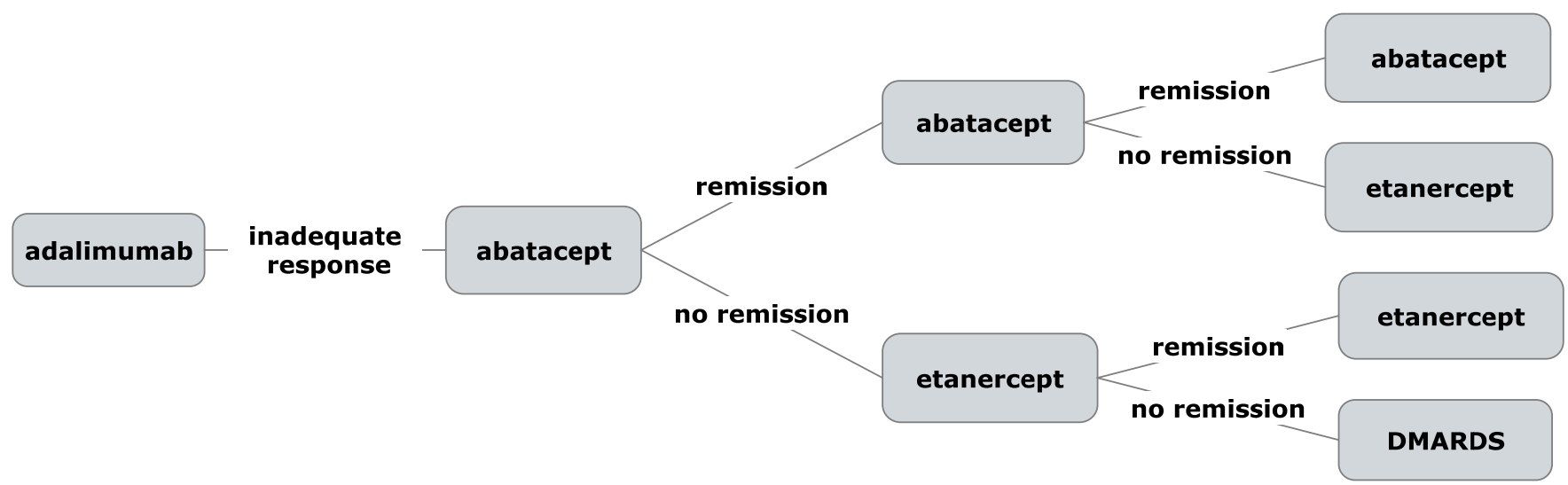

Fig. (1). Exemple of sequence A model structure with remission as effectiveness endpoint.

and consistent clinical outcomes allow to define clinical effectiveness for a given treatment more accurately and to compare across different treatment strategies for a specific patient population.

In lack of head-to-head clinical studies, which would allow to compare the clinical efficacy and cost-effectiveness of various sequential treatment strategies, modelling such comparative studies is an important and valid way to address the problem [15]. In particular, creating advanced simulation models requires expert validation of clinical assumptions and effectiveness endpoints. Achieving a state of remission or low disease activity being the therapeutic targets when treating RA, dichotomous approaches to effectiveness (success/no-success) are clinically meaningful and are generally more easily interpreted than continuous measures [4]. Success cut-off endpoints are also the standard outcomes reported in clinical trials $[5,7,9]$. However, because the DAS28 is an "ordinal score" (all degrees are not equal), it would be methodologically incorrect to calculate costeffectiveness ratios such as "cost per unit of DAS28" or "cost per unit of HAQ", even though such approaches using the HAQ have already been published [16]. Using a dichotomous approach (success/no-success =remission/no remission or LDAS/no LDAS) of the DAS28 score appeared more clinically meaningful for the purpose of this study as achieving a remission is the ultimate goal of treating RA. As both the DAS28 and HAQ scales are "ordinal" and not "cardinal" (all degrees are equal), not only effectiveness criteria expressed in "achieving remission" or "achieving LDAS" are clinically meaningful, but cost-effectiveness calculations also require fewer assumptions. This is also why such approaches appear more methodologically robust, as already published in Canada by Russel et al., [4], in France by Saraux et al., [12], in Italy by Cimmino et al., [13] and in Spain by Beresniak et al., [14].

If designed and performed correctly, simulation models allow to assess and compare the potential benefits of different sequential strategies, and to select those that appear the most cost-effective and beneficial to patients. The advantages of modelling approaches are especially salient in chronic progressive diseases where most health gains and any potential economic benefits of different treatments often become evident only in the long-term, which typically goes far beyond the scope of most clinical trials.
Concerning this model's assumptions and limitations, biologic treatments switches were allowed after 6-month intervals in case of insufficient response. While treatment switches may occur sooner in medical practice, this assumption was endorsed due to the fact that most clinical trials report effectiveness data at 6 months time points. Furthermore, the model considers that RTX potential retreatments occur at 6 -month intervals because only 6 months time points were reported in the publications at the time of model development [9]. This assumption does however imply a sustained efficacy for RTX over time, and thus, does not take into account the potential observed "flares" which may occur beyond that time point. While this assumption for RTX could be seen as overestimating the actual efficacy of RTX when compared with similar sequences including ABA, assuming RTX re-treatments at 9 months (instead of 6 months) would actually not significantly impact the model results. This is because this would concern only very few patients in the final branches of the model. Furthermore, the model did not assess the switch of one anti-TNF agent with another anti-TNF agent because anti-TNF "cycling" is not a current practice in Finland, and published scientific evidence shows that an insufficient response due to a loss of efficacy to a first anti-TNF agent is likely to lead to a similar response to a 2nd and 3rd anti-TNF agent (which is also referred to as a "class effect" because of their similar mechanism of action). Hence, Cost-effectiveness of treatment sequences using up to 2 and 3 anti-TNF agents successively were assessed in France, Spain and Italy [1214] and did not show any significant clinical improvement.

Finally, the model assumes that a potential remission or LDAS are be sustained over the 6-month treatment interval. This assumption was deemed appropriate by the expert panel at it appears to be consistent with the medical practices in Finland. In addition, as the time horizon of the model is limited to 2 years, this allows to include published clinical evidence from randomized clinical trials. Hence, as no long term effectiveness assumptions had to be made compared to published lifetime cost-effectiveness models in RA [17], this represents a significant advantage as it reduces the uncertainty of long-term assumptions. And while the relationship between DAS scores and costs is well established, it is worth noting that the DAS does not capture all aspects of Quality of Life (QOL) improvement, which 
should be considered separately to their full merit. Furthermore, as it is not the purpose of one clinical indicator to capture all dimensions of life, QOL dimensions should be collected separately using appropriate validated instruments.

Regarding the optimal criterion for cost-effectiveness assessments in RA, this has been a subject of debate and has even resulted in misleading use of concepts. Many published "cost-effectiveness" models are in fact cost-utility models, using "Quality Adjusted Life Years" (QALY) as subjective outcome measure [15,18-20]. The QALY method has received criticism, not only because different utility instruments (such as HUI, EQ5D or SF-6D questionnaires) generate statistically divergent utility scores in the same RA population, but because they can also lead to widely different cost-utility ratios [21-24]. This is raising much ethical questions, which also explains why the cost-utility (QALY) approach is now banned from key countries such as USA, Germany and France [25]. For these reasons, real cost-effectiveness analyses based on "clinically meaningful outcomes" are increasingly recommended for being methodologically more transparent, consistent and reliable for assessing novel treatments [15] and to support health care decision making [22]. In addition, it is worth noting that no significant relationship has been established between DAS and survival rates [26], as most clinical trials do not contain adequate power or follow up to differentiate mortality benefits in RA with one intervention versus another. This explains why most economic models have not included mortality as a treatment-specific consequences $[3,27]$. Because patient registries are not designed to allow any treatment comparisons, but to assess resource utilization and medical practices, effectiveness data reported in registries were not taken into account in order to avoid potential selection bias. The present results are based on published clinical data and should be updated accordingly with upcoming clinical evidence. Economic analyses in RA should also ideally consider total direct and indirect costs associated with the disease [28]. Since the costs are calculated from the public payer perspective, indirect costs, such as patient's workability, were not considered in the model. Given that RA indirect costs are considerable (i.e., loss of income due to lost work days, change in employment or salary, productivity loss, long-term disability), the overall costeffectiveness and value of different biological strategies is likely understated.

\section{CONCLUSION}

In summary, RS and LDAS are associated with lower medical costs compared to MHDAS. The results of this simulation model based on these published clinical outcomes suggest that treatment sequences of biologic agents including ABA as second biologic option are more efficacious and cost-effective than similar sequences including RTX in patients with moderate or severe RA and with an insufficient response to a first anti-TNF agent

\section{ACKNOWLEDGEMENT}

Declared none

\section{CONFLICT OF INTEREST}

This work has been supported by an unrestrictive grant from Bristol-Myers Squibb. Two authors are employees of Bristol-Myers Squibb (T. Leikola-Pelho and K. Sennfalt).

\section{REFERENCES}

[1] Puolakka K, Kautiainen H, Pohjolainen T, Virta L. Rheumatoid arthritis remains a threat to work productivity: a nationwide registerbased uncidence study from finland. Scand J Rheumatol 2010; 39: 436-8.

[2] Hyrich K, Lunt M, Watson K, Symmons D, Silman A. Register BSfRB. Outcomes after switching from one anti-tumor necrosis factor alpha agent to a second anti-tumor necrosis factor alpha agent in patients with rheumatoid arthritis: results from a large UK national cohort study. Arthritis Rheum 2007; 56: 13-20.

[3] Gabriel S, Drummond M, Maetzel A, et al. Omeract 6 economics working group report: a proposal for a reference case for economic evaluation in rheumatoid arthritis. J Rheumatol 2003; 30: 887-90.

[4] Russell A, Beresniak A, Bessette L, et al. Cost-effectiveness modeling of abatacept versus other biologic agents in dmards and anti-tnf inadequate responders for the management of moderate to severe rheumatoid arthritis. Clin Rheumatol 2008; 28(4): 403-12.

[5] Genovese M, Schiff M, Luggen M, et al. Efficacy and safety of the selective co-stimulation modulator abatacept following 2 years of treatment in patients with rheumatoid arthritis and an inadequate response to anti-tumour necrosis factor therapy. Ann Rheum Dis 2008; 67: 547-54.

[6] Genovese M, Becker J, Schiff M, et al. Abatacept for rheumatoid arthritis refractory to tumor necrosis factor alpha inhibition. N Engl J Med 2005; 353: 1114-23.

[7] Bombardieri S, Ruiz A, Fardellone P, et al. Effectiveness of adalimumab for rheumatoid arthritis in patients with a history of tnf-antagonist therapy in clinical practice. Rheumatology 2007; 46: 1191-9.

[8] Cohen S, Emery P, Greenwald M, et al. Rituximab for rheumatoid arthritis refractory to anti-tumor necrosis factor therapy: results of a multicenter, randomized, double-blind, placebo-controlled, phase iii trial evaluating primary efficacy and safety at twenty-four weeks. Arthritis Rheum 2006; 54: 2793-806.

[9] Keystone E, Fleischmann R, Emery P, et al. Safety and efficacy of additional courses of rituximab in patients with active rheumatoid arthritis. Arthritis Rheum 2007; 56: 3896-908.

[10] Karlson J, Kristensen L, Kapetanovic M, Gulfe A, Saxne T, Geborck P. Treatment response to a second or third tnf-inhibitor in ra: Results from the south swedish arthritis treatment group register. Rheumatology 2008; 47: 507-13.

[11] Beresniak A, Gossec L, Goupille P, et al. Direct cost-modeling of rheumatoid arthritis according to disease activity categories in France. J Rheumatol 2011; 38(3): 439-45.

[12] Saraux A, Gossec L, Goupille $\mathrm{P}$, et al. Cost-effectiveness modelling of biological treatment sequences in moderate to severe rheumatoid arthritis in france. Rheumatology (Oxford) 2010; 49: 733-40.

[13] Cimmino M, Leardini G, Salaffi F, et al. Assessing the costeffectiveness of biologic agents for the management of moderateto-severe rheumatoid arthritis in anti-tnf inadequate responders in italy: A modelling approach. Clin Exp Rheumatol 2011; 29(4): 633-41.

[14] Beresniak A, Ariza-Ariza R, Garcia-Llorente J, Ramirez-Arellano A, Dupont D. Modelling cost-effectiveness of biologic treatments based on disease activity scores for the management of rheumatoid arthritis in Spain. Int J Inflam 2011; 2011; 727634.

[15] Beresniak A, Russell A, Haraoui B, Bessette L, Bombardier C, Duru G. Advantages and limitations of utility assessment methods in rheumatoid arthritis. J Rheumatol 2007; 34: 2193-200.

[16] Osiri M, Kamolratanakul P, Maetzel A, Tugwell P. Cost effectiveness analysis of disease modifying antirheumatic drugs in rheumatoid arthritis. Rheumatol Int 2007; 27: 1063-9.

[17] Davies A, Cifaldi M, Segurado O, Weisman M. Cost-effectiveness of sequential therapy with tumor necrosis factor antagonists in early rheumatoid arthritis. J Rheumatol 2009; 36: 16-26.

[18] Kielhorn A, Porter D, Diamantopoulos A, Lewis G. Uk cost-utility analysis of rituximab in patients with rheumatoid arthritis that failed to respond adequately to a biologic disease-modifying antirheumatic drug. Curr Med Res Opin 2008; 24: 2639-50.

[19] Vera-Llonch M, Massarotti E, Wolfe F, et al. Cost-effectiveness of abatacept in patients with moderately to severely active rheumatoid arthritis and inadequate response to tumor necrosis factor-alpha antagonists. J Rheumatol 2008; 35: 1745-53. 
[20] Wailoo A, Bansback N, Brennan A, Michaud K, Nixon R, Wolfe F. Biologic drugs for rheumatoid arthritis in the medicare program: A cost-effectiveness analysis. Arthritis Rheum 2008; 58: 939-46.

[21] Duru G, Auray JP, Beresniak A, Lamure M, Paine A, Nicoloyannis N. Limitations of the methods used for calculating quality-adjusted life-year values. Pharmacoeconomics 2002; 20: 463-73.

[22] Mc Gregor M, Caro J. Qalys: are they helpful to decision makers? Pharmacoeconomics 2006; 24: 947-52.

[23] Marra C, Woolcott J, Kopec J, et al. A comparison of generic, indirect utility measures (the hui2, hui3, sf-6d and the eq-5d) and disease-specific instruments (the raqol and the haq) in rheumatoid arthritis. Soc Sci Med 2005; 60: 1571-82.
[24] Conner-Spady B, Suarez-Almazor M. Variation in the estimation of quality adjusted life-years by different preference-based instruments. Med Care 2003; 41: 791-801.

[25] Neumann PJ, Weinstein MC. Legislating against use of costeffectiveness information. N Engl J Med 2010; 363: 1495-97.

[26] Bansback N, Ara R, Karnon J, Anis A. Economic evaluations in rheumatoid arthritis: a critical review of measures used to define health states. Pharmacoeconomics 2008; 26: 395-408.

[27] Maetzel A, Tugwell P, Boers M, et al. Economic evaluation of programs or interventions in the management of rheumatoid arthritis: Defining a reference case. J Rheumatol 2003; 30: 891-6.

[28] Emery P. Review of health economics modelling in rheumatoid arthritis. Pharmacoeconomics 2004; 22: 55-69.

(C) Puolakka et al.; Licensee Bentham Open.

This is an open access article licensed under the terms of the Creative Commons Attribution Non-Commercial License (http://creativecommons.org/licenses/by-nc/ $3.0 /$ ) which permits unrestricted, non-commercial use, distribution and reproduction in any medium, provided the work is properly cited. 